\title{
Base-specific pre-melting and melting transitions of DNA in presence of ionic liquids probed by synchrotron-based UV Resonance Raman scattering
}

Cettina Bottari ${ }^{1,2}$, Sara Catalini ${ }^{3}$, Paolo Foggi ${ }^{3,4}$, Ines Mancini ${ }^{5}$, Andrea Mele ${ }^{6}$, Diego Romano Perinelli $^{7}$, Alessandro Paciaroni ${ }^{8}$, Alessandro Gessini ${ }^{1}$, Claudio Masciovecchio ${ }^{1}$, Barbara $\operatorname{Rossi}^{1,5^{*}}$

1. Elettra-Sincrotrone Trieste, S.S. 114 km 163.5, Basovizza, 34149, Trieste, Italy

2. Department of Physics, University of Trieste, Via A. Valerio 2, 34127, Trieste, Italy

3. European Laboratory for Non-Linear Spectroscopy, LENS, Via Nello Carrara, 1, 50019, Sesto Fiorentino, Firenze, Italy

4. Department of Chmistry, Biology and Biotechnology, University of Perugia, Via Elce di Sotto 8, I-06123 Perugia, Italy

5. Department of Physics, University of Trento, Via Sommarive, 14 - 38123 Povo Trento, Italy

6. Department of Chemistry, Materials and Chemical Engineering ' G. Natta', Politecnico di Milano and INSTM Local Unit, Piazza L. da Vinci 32, 20133, Milano, Italy

7. School of Pharmacy, University of Camerino, Via Gentile III da Varano, Camerino (MC) 62032, Italy

8. Department of Physics and Geology, University of Perugia, 06123, Perugia, Italy

*Correspondence to: barbara.rossi@elettra.eu

\begin{abstract}
Hydrated ionic liquids (ILs) have been identified as solvent media able to enhance the structural stability of deoxyribonucleic acid (DNA). In this work, we investigate the molecular interaction between imidazolium-based ILs and DNA during its thermal unfolding pathway, by exploiting synchrotron-based UV Resonance Raman scattering (UVRR) experiments. This technique gives a selective focus on the thermal responses of specific nucleobases in the structure of DNA providing the experimental sensitivity to both cooperative and local structural changes occurring along the complex unfolding process of DNA. UVRR measurements probe two distinct temperature-dependent phenomena occurring in the DNA double-helix, i.e. a non-cooperative pre-melting mainly involving adenine bases and a cooperative melting transition primarily localized on guanine tracts. The analysis of Raman spectra reveals that both the cation and anion of the ionic liquids strongly interact with the structure of DNA, thus affecting the melting process but not perturbing the pre-melting transition that precedes the complete separation of the strands of DNA. Overall these results suggest that the dominant interaction occurs between the imidazolium cation and the bases of guanine and thymine in the structure of DNA, in agreement with previous results of molecular dynamics simulations.
\end{abstract}

Keywords: UV Resonance Raman, DNA, melting, pre-melting, ionic liquids 


\section{Introduction}

From several years, ionic liquids (ILs) continue to attract a great deal of attention thanks to their fascinating properties of vanishing vapour pressure, good thermal and chemical stability, tunable polarity and solubility, high ion density and ionic conductivity [1,2]. Several applications of ILs have been explored in various fields, including organic synthesis [3], electrochemical devices [4], photochemical cells [5, 6] and catalysis [7]. The numerous possible combinations of cations and anions have made ILs attractive alternatives for replacing the traditional organic solvents and aqueous media in many types of reactions $[8,9]$. Since the first report on the use of ILs to increase the activity of enzyme [10], ILs have attracted a particular interest as new and highly efficient solvents or co-solvents to be used in the life science. Several chemical-physical investigations on the interaction between ILs and saccharides, membranes, peptides/proteins and nucleic acid have demonstrated that ILs offer unique opportunities as alternatives solvents for improving stability and activity of bio-molecules [11-14].

Nucleic acids such as DNA and RNA are anionic polyelectrolytes that are usually considered stable in aqueous solution, although various conditions of temperature, $\mathrm{pH}$, ionic strength and solvent properties can disrupt their typical helix structure, leading to denaturation. A first experimental observation of unusual long-term structural and chemical stability of DNA stored at room temperature in hydrated choline-based ILs was provided by Vijayaraghavan et al. [15]. A number of other publications have reported on the effect of various classes of ILs and deep eutectic solvents (DES) on the solvation properties, stability and packing of DNA [16-19] using both experimental and theoretical techniques. Several studies suggest that ionic liquids with cations based on imidazolium are good co-solvents able to improve the long-term and thermal stability of DNA [20-24]. The molecular mechanism of the interaction between this class of ILs and DNA has been extensively investigated by different authors [20-24] but there is not a fully agreement on the driving forces that dominate the binding mechanism. Molecular dynamics simulations and spectroscopic experiments unraveled that DNA maintains the native Bconformation in hydrated ILs [20,25] mainly thanks to the electrostatic interactions of the imidazolium cations of IL with the negatively charged backbone of DNA. In addition, the groove binding mechanism of cations through hydrophobic and polar forces seems to contribute significantly to DNA stability [20]. Experimental measurements on DNA dissolved in 1-butyl-3methylimidazolium chloride [BMIM]Cl indicate that hydrophobic interactions between the hydrocarbon chains of imidazolium-based cations and the bases of DNA are the dominant driving force in the binding mechanism [21]. This view has been confirmed also by ultrafast molecular rotor experiments [24]. Other investigations on DNA stability in the presence of [BMIM]Cl suggest that the electrostatic attraction between the charge groups of cations and the DNA phosphate moieties is the dominant force responsible of the stabilizing the DNA [22,26,27]. Conversely, a more recent study [23] hypothesizes that DNA interacts with imidazolium-based ILs through an intercalation binding mode of the cation and this interaction becomes stronger as the alkyl chain length increased. Although molecular dynamics simulations infer that the cation species play the lead role in the interaction mechanism between DNA and ILs [20], the effect of different kind of anions on the stability of DNS has not been widely explored. However, evidence has been reported that for some imidazolium-based ILs the anions tend to establish a greater number of hydrogen bonds with the nucleobases of DNA than the cations [17].

In the present work, we focus on the effect exerted by imidazolium-based ILs on the pathway of DNA melting, using synchrotron-based UV Resonance Raman scattering (UVRR) [28]. Detecting the nature of the melting transition in double-strand DNA requires experimental sensitivity to both cooperative and local structural changes, since DNA thermally unravels passing through intermediate denatured states. Promoted by the increment of the temperature, the 
hydrogen bond breaks and the DNA strands separate base by base leading to a complex melting pathway.

Respect to the off-resonance Raman technique, UVRR spectroscopy offers the unique structural specificity associated with the molecular vibrational frequencies together with the high sensitivity and selectivity provided by the resonance enhancement [29-31]. Additionally, the tunability of UV synchrotron radiation source permits a fine matching between the excitation wavelength and the electronic transitions of the nucleobases. This ensures to selectively isolate in the UVRR spectra of DNA the contributions arising from individual bases [28]. Synchrotronbased UVRR technique is a powerful tool for efficiently probing in DNA the structural changes associated with base pairing and stacking interactions and the alteration in the hydrogen-bond at the ring sites [28-32]. The effectiveness of this experimental approach is validated in the present work where Raman markers sensitive to the following factors are proposed: i) base stacking and ii) hydrogen bonds interactions selectively involving guanine, adenine and thymine. This allows to distinguish the effects exerted by ILs on the DNA melting process and on the structural perturbations that precede the onset of strand separation, i.e. pre-melting transitions. Insights on the binding mechanism between ILs and DNA along the thermal denaturation pathway are obtained by the analysis of precise spectral signals recognized in the UVRR profiles. Complementary experiments of UV absorption, circular dichroism and micro differential scanning calorimetry support the results of UVRR experiments.

\section{Materials and methods}

\section{Sample preparation}

DNA sodium salt from salmon testes (sDNA) (CAS number 438545

MW= $1.310^{6}$ Da, \% G-C content: 41.2\%) was purchased by Sigma

-062000 base-pairs, further purification. The absence of proteins as contamination was checked by measuring the absorbance ratio of a DNA solution at 260 and $280 \mathrm{~nm}$ that was found to be 1.9. Lyophilized powder of sDNA was dissolved in phosphate-buffered saline $10 \mathrm{mM} \mathrm{PBS}$ at $\mathrm{pH}=7.4$ and gently stirring for $24 \mathrm{~h}$ to achieve a limpid solution.

Deoxynucleotide (dNTP) set composed by: 2'-deoxyadenosine 5'- triphosphate sodium salt solution (dATP), 2'-deoxycytidine 5'- triphosphate disodium salt (dCTP), 2'-Deoxyguanosine 5'triphosphate trisodium salt solution (dGTP) and thymidine $5^{\prime}$ - triphosphate sodium salt solution (dTTP) at $100 \mathrm{mM}$ was purchased by Sigma-Aldrich. For UVRR measurements, each dNTP was diluted in PBS at pH 7.4 in order to reach the final concentration of $5 \mathrm{mM}$.

Figure 1 reports the chemical structures of ILs that we have considered in the present study. 


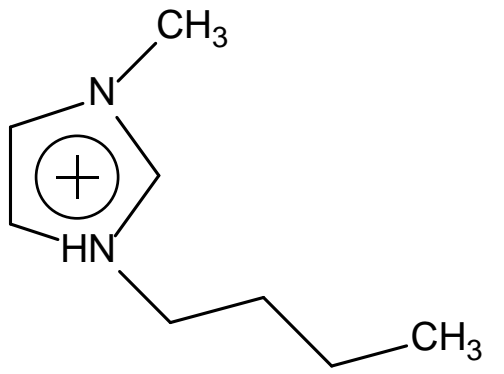

1-butyl-3-methylimidazolium [BMIM]

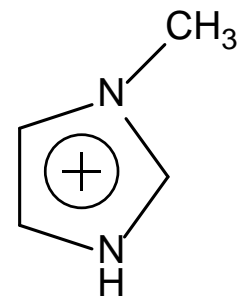

1-methylimidazolium [MIM]<smiles>COS(=O)(=O)[O-]</smiles>

methyl sulfate $\mathrm{MeSO}_{4}{ }^{-}$

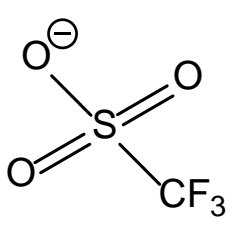

triflate TfO

Figure 1: Chemical structures of the ILs considered in the present study: [BMIM] as methyl sulfate or triflate, and [MIM] triflate.

All the ionic liquids 1-butyl-3-methylimidazolium triflate [BMIM]TfO, 1-butyl-3methylimidazolium methyl sulfate [BMIM] $\mathrm{MeSO}_{4}$ and 1-methylimidazolium triflate [MIM]TfO were purchased by IoLiTec with a purity of 99\%. Before the measurements, all the ILs were dried inside a desiccator under vacuum with phosphorus pentoxide for $48 \mathrm{~h}$ in order to remove any water contamination.

For UVRR experiments, solutions at concentration of sDNA equal to $0.2 \mu \mathrm{M}$ in BPB at $\mathrm{pH}=7.4$ were prepared. For the preparation of DNA/IL samples, each ionic liquid was added to the solution of sDNA to reach the final concentration of $62 \mathrm{mM}$ of IL in PBS, corresponding to a stoichiometric ratio of sDNA/IL of $0.00310^{-3}$. All the solutions were freshly prepared for UVRR measurements and they appeared limpid before the running of experiments and after the thermal heating.

The solutions of sDNA with and without IL used for UV absorbance, circular dichroism and calorimetry experiments were diluted 8 times in PBS with respect to the samples prepared for UVRR measurements.

\section{UVRR experiments}

UVRR spectra were collected using the synchrotron (SR)-based UVRR set-up available at the BL10.2-IUVS beamline of Elettra Sincrotrone Trieste (Italy) [28,32]. The exciting wavelengths was set at 250 and $266 \mathrm{~nm}$ for the experiments reported here. All UVRR spectra were recorded in back-scattered geometry by using a single pass of a Czerny-Turner spectrometer (Trivista 557, Princeton Instruments, $750 \mathrm{~mm}$ of focal length) equipped with holographic grating at $1800 \mathrm{~g} / \mathrm{mm}$. The spectral resolution was set at 1.7 and $1.9 \mathrm{~cm}^{-1} /$ pixel for the spectra collected with 266 and $250 \mathrm{~nm}$ of excitation wavelength, respectively. The calibration of the spectrometer was standardized using cyclohexane (spectroscopic grade, Sigma Aldrich). The radiation power on the samples was kept maximum of $200 \mu \mathrm{W}$. Any possible photo-damage effect due to a prolonged exposure of the sample to UV radiation was avoided by continuously spinning the sample cell during the measurements. The UVRR spectra were collected for each sample in the 
temperature range between 291-375 $\mathrm{K}$ with $\Delta \mathrm{T}=3$ and $\Delta \mathrm{T}=4 \mathrm{~K}$ for the measurements with 266 and $250 \mathrm{~nm}$ of excitation wavelength, respectively.

Circular Dichroism Spectroscopy

Circular dichroism (CD) spectra were recorded using Jasco J-710 polarimeter equipped with a thermal bath for controlling the temperature of the samples during the measurements. All the solutions were measured from 305 to $368 \mathrm{~K}$ in a temperature-controlled quartz cell of $10 \mathrm{~mm}$ path-length. Each CD spectrum was averaged over 10 scans recorded in the range from 220 to $320 \mathrm{~nm}$, at a scanning speed of $100 \mathrm{~nm} / \mathrm{min}$ and $1 \mathrm{~nm}$ of bandwidth. Measurements were performed under a constant nitrogen flow, which was used to purge the ozone generated by the light source of the instrument.

\section{UV/Vis Spectroscopy}

UV Absorption measurements were recorded with a PerkinElmer, model Lambda 25 UV/Vis spectrometer at double beam equipped with a Peltier thermostattable for controlling the temperature of the samples. All the solutions were measured from 305 to $370 \mathrm{~K}$ in a rectangular quartz cell of 10mm path-length. The spectra were recorded in the range from 190 to $600 \mathrm{~nm}$ at a scanning speed of $120 \mathrm{~nm} / \mathrm{min}$ and $1 \mathrm{~nm}$ of bandwidth.

MicroDifferential Scanning Calorimetric (mDSC) experiments

Thermograms of sDNA solution alone or in presence of IL were recorded using a microDSC III (Setaram, France). $0.750 \mathrm{~g}$ of the sample and an equal amount of PBS as reference were filled in Hallostey calorimetric cells. Samples were equilibrated for $20 \mathrm{~min}$ at the starting temperature of $293 \mathrm{~K}$ before performing a heating ramp in the temperature range $293-373 \mathrm{~K}$ at a rate of $0.5 \mathrm{~K}$ /min. The temperature $\left(\mathrm{T}_{\mathrm{m}}, \mathrm{K}\right)$ and the calorimetric enthalpy $(\Delta \mathrm{H}, \mathrm{kJ} / \mathrm{mol})$ were calculated from the peak and the area of the transition, respectively using the tangent method

\section{Results}

UV Resonance Raman conditions and assignments of main Raman bands of DNA

Figure 2(a)-(b) show the experimental UVRR spectra collected on equimolar solutions of the pure deoxynucleotides dTNP using 250 and $266 \mathrm{~nm}$ as excitation wavelengths, respectively. Attention is focused on the wavenumber region between 1150 and $1750 \mathrm{~cm}^{-1}$ where the main signals associated to DNA nucleobases are found. The comparison between the Raman spectra of the pure dTNP with that of sDNA (Fig. 2(c)-(d)) is useful to identify the contribution of each individual nitrogenous bases in the complex spectrum of DNA obtained with the corresponding excitation wavelength. The intensity of the spectra of the pure dTNP in Fig. 2 have been rescaled accordingly to their relative abundance in the sequence of sDNA (41.2 \% G-C and $58.8 \%$ A-T pairs). A relative good resemblance is found between the UVRR spectra of sDNA (Fig. 2(a) and (d)) and the profiles obtained as the sum of the scaled spectra of single dNTP (Fig. 2(e)-(f)) both for the excitation wavelengths at 250 and $266 \mathrm{~nm}$. The slight differences observed between experimental and reconstructed spectra can be probably attributed to the different conformation assumed by sugar-phosphate skeleton in DNA strand with respect to pure dNTP and to the stacking interactions among bases in the duplex helix structure [33]. 


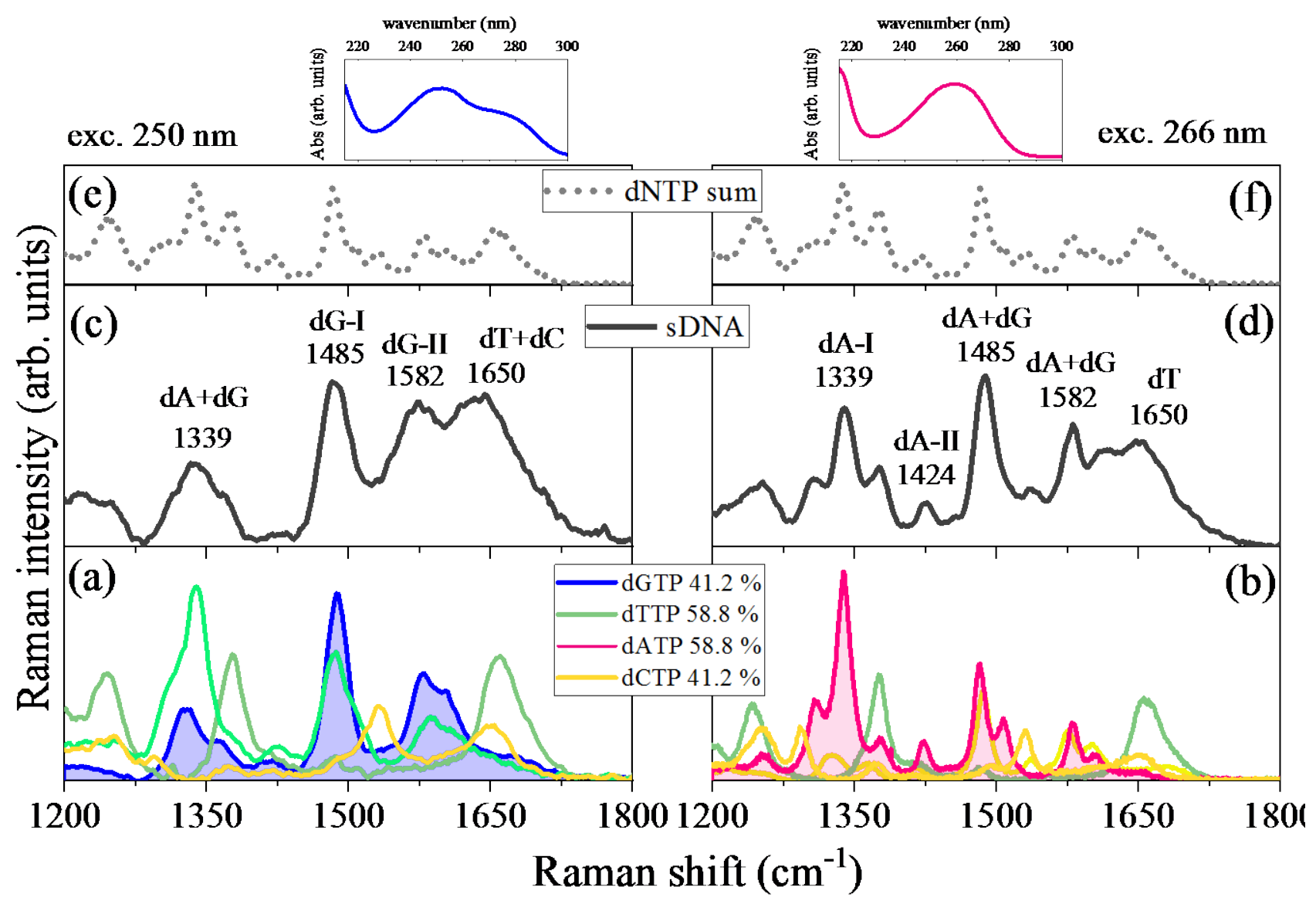

Figure 2: UVRR spectra of deoxynucleotides dNTP and of sDNA collected using excitation wavelengths at $250 \mathrm{~nm}$ (a)-(c) and $266 \mathrm{~nm}$ (b)-(d), respectively; the intensity of each spectrum of dNTP has been rescaled on the basis of its percentage content in the sequence of sDNA. (e)-(f): profiles obtained as sum of the individual spectra of dNTP. Insets at the tops: UV absorbance spectra of dGTP and dATP (left and right panel, respectively).

Excitation wavelengths in the UV range yields to Raman bands of the highest intensity, especially for the vibrational signals arising from the nucleic acid bases [34]. Sugar and phosphate group vibrations are practically negligible from UVRR spectra of sDNA collected with 250 and $266 \mathrm{~nm}$ as excitation wavelengths, as well as any other interfering signal attributable to the solvent.

The UV-Vis absorption spectra of the deoxynucleotides dGTP and dATP are reported in the insets of Fig. 2. The spectrum of both dGTP and dATP is characterized by a broad absorption profile in the region between 230 and $280 \mathrm{~nm}$, which is generated by two $\pi-\pi^{*}$ electronic transitions allowed for both guanine $(\mathrm{dG})$ and adenine $(\mathrm{dA})$ residues. The absorption band centered at $\sim 255 \mathrm{~nm}$ is assigned to the electronic transition mostly localized on the N7=C8 double bond of the imidazole ring. The lowest-energy absorption band at $\sim 275 \mathrm{~nm}$ is associated with the transition involving the triene fragment C2=N3-C4=C5-N7=C8 [30].

Fig. 2(a)-(c) shows that the excitation wavelength at $250 \mathrm{~nm}$ well overlaps with the energy of the electronic transition localized on the double bond N7 = C8 of the imidazole ring, thus satisfying a better resonance condition for dG residue than dA [30]. Likewise, with 266-nm as excitation wavelength, resonance Raman bands assigned to the adenine $\mathrm{dA}$ residue mostly contribute to the spectrum of sDNA (Fig. 2(d)). 
In the wavenumber range $1200-1800 \mathrm{~cm}^{-1}$ various bands of sDNA assigned to purine and pyrimidine vibrations are responsive to the changes occurring in the ring electronic structure. These signals exhibit a strong sensitivity to base unstacking and to alterations in the hydrogenbonding at ring sites. Table S1 in the SI section reports the detailed assignment of the normal modes composition for the main spectral features observed in the UVRR profiles of sDNA obtained with 250 and $266 \mathrm{~nm}$ as excitation wavelengths.

The Raman band at $\sim 1339 \mathrm{~cm}^{-1}$ in the spectrum of sDNA obtained with $266 \mathrm{~nm}$ as excitation wavelength is mainly assignable to the vibration mode labelled as dA-I arising from the coupled stretching vibrations of N7=C8 and C5- N7 bonds of adenine [30,35,36]. This mode is a good marker of the adenine nucleoside conformation [37] and of the hydrogen-bonding state at the acceptor site N7 of adenine [38,39]. Such a vibrational mode can be well isolated in the spectrum of DNA using $266 \mathrm{~nm}$ as excitation wavelength. In the $250 \mathrm{~nm}$-excited spectrum of sDNA (Fig. 2(c)) the $d A$ signal at $\sim 1339 \mathrm{~cm}^{-1}$ is strongly overlapped with the vibration arising from the N7=C8 and C8-N9 ring stretching plus C8-H in-plane bending of dG [30,34]. The $266 \mathrm{~nm}$ excited spectrum of sDNA shows another signal at $\sim 1424 \mathrm{~cm}^{-1}$ associated with the adenine residue, labelled as dA-II. This band is attributed to a combination of stretching mode of C4-N9 and bending of C8-H bonds. The changes in the frequency position of the mode dA-II reflect the modification of hydrogen-bonds at the C6- $\mathrm{NH}_{2}$ site of adenine [31, 36].

The prominent Raman band at $\sim 1485 \mathrm{~cm}^{-1}$ is associated to vibrational modes that involve the N7=C8 and C8-N9 ring stretching coupled with C8-H in-plane deformation of purine group [30,34,40,41]. The peak at $\sim 1582 \mathrm{~cm}^{-1}$ is assigned to N3-C4, C4-C5, and C5-N7 stretching motions $[34,39,42]$ of the same purine moiety. Both these modes show marked Raman intensity hypochromism effects upon the formation of stacked or ordered nucleic base structures [41-43] and for this reason they are sensitive structural markers for DNA. In the 266 nm-excited spectra of sDNA (Fig. 2(d)) the signals at $\sim 1485 \mathrm{~cm}^{-1}$ and $1582 \mathrm{~cm}^{-1}$ contain comparable contributions arising from dA and dG residues. Conversely, at the excitation wavelength of $250 \mathrm{~nm}$, there is an appreciable enhancement of the modes labelled as dG-I and dG-II, associated to the vibrations localized on the purine ring of dG residue (Fig. 2(c)). This is consistent with the proximity of the excitation energy to the 255-nm transition of dG that largely involves the N7=C8 double bond of the imidazole ring [44]. In the spectrum of Fig. 2(c), the large bump centered at $\sim 1650 \mathrm{~cm}^{-1}$ is the result of the superposition of vibrational modes localized on thymine (dT) and cytosine (dC) residues. Consistently with the electonic transition observed for $\mathrm{dT}$ at $\sim 265 \mathrm{~nm}$, the contribution of dT to the mode at $1650 \mathrm{~cm}^{-1}$ is slightly enhanced in the $266 \mathrm{~nm}$-excited spectra of sDNA (Fig. 2(d)) with respect to the profiles collected at $250 \mathrm{~nm}$ of excitation wavelenght. The Raman band labelled as $\mathrm{dT}$ is probably related to a coupled stretching of $\mathrm{C} 4=\mathrm{O}$ and $\mathrm{C} 5=\mathrm{C} 6$ bonds of the dT residue $[45,46]$. Despite the relative complexity of this stretching vibration, the large localization of the mode on the $\mathrm{C} 4=\mathrm{O}$ group makes the signal at $\sim 1650 \mathrm{~cm}^{-1}$ considerably sensitive to any perturbations occurring at that site of thymine residue [47].

\section{Temperature-dependence of UVRR spectra of DNA in presence of ILs}

Figure 3(a)-(d) show the temperature-evolution of UV Raman spectra, collected using 250 and $266 \mathrm{~nm}$ as excitation wavelengths, for sDNA in PBS and in hydrated [BMIM]TfO, as an example. The comparison with the profiles of the hydrated ionic liquid (dashed lines in Fig. 3(a)(d)) allows to clearly separate the Raman signals arising from sDNA from those associated to vibrations of the IL. 


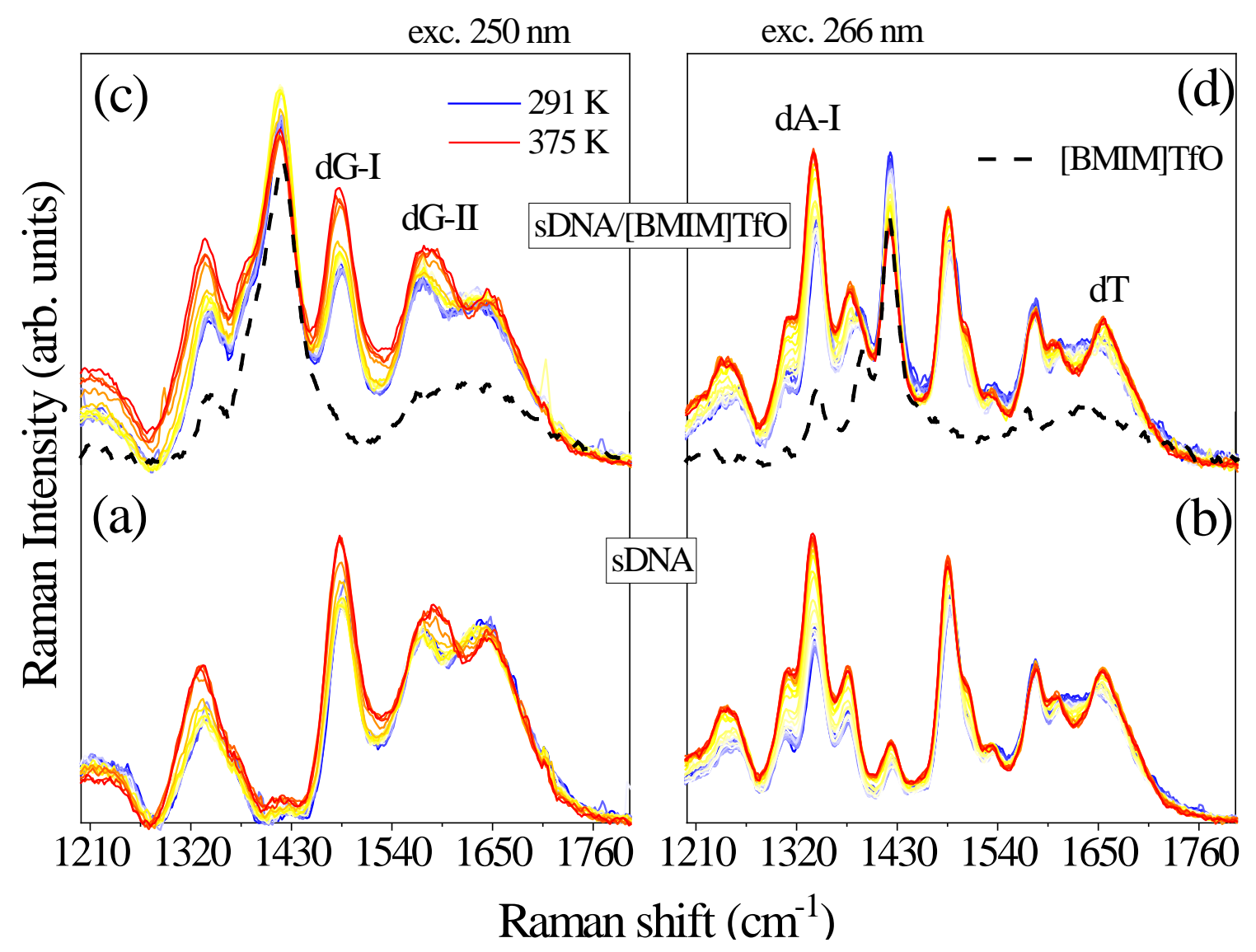

Figure 3: Temperature-dependent UVRR spectra of sDNA in PBS and in hydrated [BMIM]TfO obtained with excitation wavelengths at $250 \mathrm{~nm}$ (a)-(c) and $266 \mathrm{~nm}$ (b)-(d), respectively. The dashed lines represent the spectra of hydrated [BMIM]TfO.

Fig. 3(a)-(b) show that, as the temperature is increased from 291 to $375 \mathrm{~K}$, the intensity of the signals dG-I, dG-II, dA-I and dT of sDNA increases substantially with a concomitant shift in the frequency position of these bands. The intensity recovery of the modes dG-I, dG-II and dA during thermal denaturation is largely attributed to the removal of the attenuation effect of the stacked bases on their nearest neighbors as the DNA melts, i.e. the so-called Raman hyperchromic effect $[34,37,40,41,43,47,48]$. The enhancement of the UV absorbance of DNA upon thermal denaturation - which in turn leads to the enhancement of the resonance of aromatic ring of nucleotides - has been related to two effects: i) the disruption of vertical base-base stacking interactions and ii) the perturbation of hydrogen bonds (HB) between complementary bases in the double strand of DNA [40,49,50]. The different temperature-dependent behavior observed for the intensity of the modes dG-I and dA-I in the spectra of Fig. 3 suggests dissimilar staking arrangements involving the residues of guanine and adenine at different stages of the melting pathway. In addition, the frequency shifts of the bands dG-I, dA-I and dT upon thermal unfolding of DNA possibly reflect the perturbation of hydrogen bonding states of the base residues of guanine, adenine and thymine [35-38,40,41]. The detailed analysis of the multiwavelength excited UVRR spectra of DNA allows to extract quantitative information on the intermediate and melted state of DNA with a special focus on the structural changes localized on guanine, adenine and thymine tracts of DNA sequence.

\section{Discussion}


Effect of ILs on cooperative melting transformation localized on dG tracts

Figure 4(a)-(d) report the relative intensities as a function of temperature of the Raman band dGI at $\sim 1485 \mathrm{~cm}^{-1}$ for sDNA pristine and in the presence of the ionic liquids [BMIM]TfO, [MIM]TfO and [BMIM]MeSO 4 . All the plots have been normalized to $(0,1)$ for a better comparison. The Raman profiles of Figure 4 reflect the structural changes primarily localized in the DNA tracts of guanine bases as a function of increasing temperature.

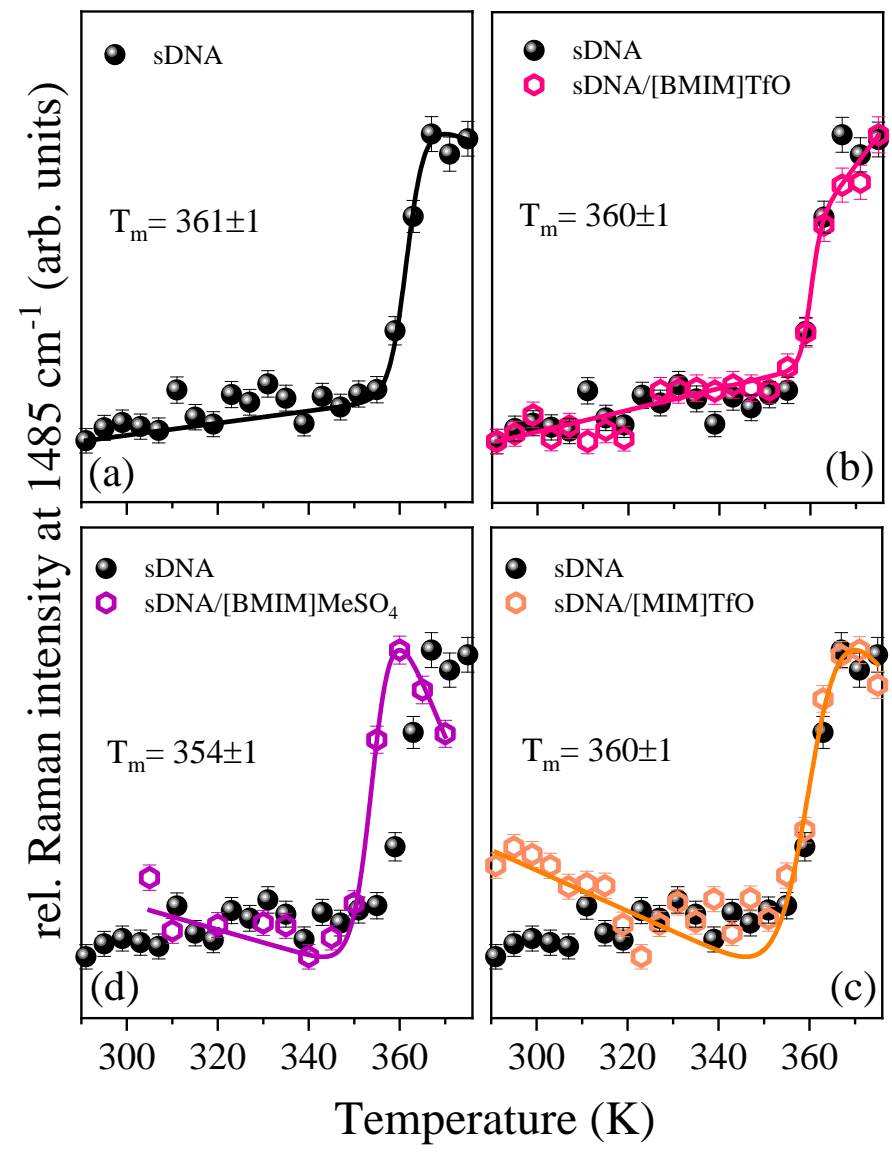

Figure 4: Temperature-dependent relative UVRR intensities of dG-I at $\sim 1485 \mathrm{~cm}^{-1}$ for sDNA (a) and sDNA in the presence of [BMIM]TfO (b), [MIM]TfO (c) and [BMIM] $\mathrm{MeSO}_{4}(\mathrm{~d})$. Continuous lines are fitting of the experimental data by using eqn. (1), see details in the text. The melting temperatures $T_{m}$ reported in the panels for the different systems have been estimated as described in the text.

The intensity of dG-I for sDNA (Fig. 4(a)) increases gradually between 290 and $355 \mathrm{~K}$ and then it exhibits a sharp increase at the correspondence to the value of $\sim 360 \mathrm{~K}$ that defines the melting transition temperature $T_{m}$ characteristic of sDNA. The trend observed in Fig. 4(a) is typical of a two-state transition and it suggests the presence of a cooperative structural transformation involving the guanine-pairs during the melting process. The sudden recovery of the Raman hyperchromicity occurring at $T_{m} \sim 360 \mathrm{~K}$ reflects the unstacking of guanine bases occurring during the strand separation and the full denaturation of the double-stranded structure of DNA [37, 42, 43, 47]. No significant changes in the intensity of the dG-I band of sDNA are detected for temperatures above the $\mathrm{T}_{\mathrm{m}}$. The data indicate that no further observable structural modifications in the secondary structure of sDNA occur once strand separation is complete, in agreement with the results obtained also on single-stranded polynucleotides sequences [37]. A 
similar temperature behavior is found also for the intensity of the band dG-II at $\sim 1582 \mathrm{~cm}^{-1}$ that exhibits the same sharp increment at the melting temperature (see Figure S1 in the SI section). This supports the conclusion that in the 250 nm-excited UVRR spectra of DNA the two modes dG-I and dG-II can be used as markers for the cooperative base-stacking interactions of guanine bases during the melting transition of DNA.

Quite interestingly, the plots in Fig. 4 evidence a different effect operated by the ILs on the thermal unfolding pathway of DNA. For the case of [BMIM]TfO and [MIM]TfO (Fig. 4(b)-(c)), the similar temperature-dependence of the intensity of dG-I band in the presence and absence of the ILs suggests that the relative reduction in the extent of base stacking interactions along the all stages of the melting transition is comparable. In the presence of [BMIM] $\mathrm{MeSO}_{4}$, the melting profile of DNA shows a notable decrement in the melting temperature, as evident by looking to the Fig. 4(c). This is consistent with the predicted interaction between the cations of IL and the backbone and grooves of DNA, as suggested by molecular dynamics simulations and experimental measurements [20-27].

As stated before, the temperature profiles reported in Fig. 4 can be reasonably approximated with a two-state transition law, as proposed by L. Movileanu et al. [51] for the determination of the bases contribution to the thermodynamics of melting transitions in DNA. The Raman intensities $I(T)$ of the band dG-I reported in Fig. 4 have been fitted by using the eqn (1):

$\mathrm{I}(T)=\frac{\mathrm{I}_{\mathrm{D}}+\mathrm{m}_{\mathrm{D}} \cdot \mathrm{T}+\left(\mathrm{I}_{N}+\mathrm{m}_{\mathrm{N}} \cdot \mathrm{T}\right) \cdot \mathrm{e}^{\frac{\Delta \mathrm{H}_{\mathrm{vib}}}{\mathrm{R}} \cdot\left(\frac{1}{\mathrm{~T}_{\mathrm{m}}}-\frac{1}{\mathrm{~T}}\right)}}{1+\mathrm{e}^{\frac{\Delta \mathrm{H}_{\mathrm{vib}}}{\mathrm{R}} \cdot\left(\frac{1}{\mathrm{~T}_{\mathrm{m}}}-\frac{1}{\mathrm{~T}}\right)}}$

In eqn (1), $I_{N}$ and $I_{D}$ represent the intensities of the native and denaturated state of DNA, respectively, $\mathrm{R}$ is the gas constant, $\Delta H_{v i b}$ and $T_{m}$ are the enthalpy variation and the melting temperature associated to the process. The parameters $m_{D}$ and $m_{N}$ describe the linear temperature-dependence of the band intensity in the pre and post-melting regions, respectively. Fig. 4 shows that the temperature-dependence of the dG-I band is fairly satisfactorily reproduced by eqn (1). The data fitting allows one to quantitatively determine the melting temperature $T_{m}$ associated with the unstacking of guanine bases with good precision. The results are summarized in Table 1.

Table 1: Melting temperature $T_{m}$ of sDNA with and without ILs. $T_{m}$ values are obtained by fitting of the experimental data of Fig 4 with eqn (1).

\begin{tabular}{|l|c|}
\hline & \multicolumn{1}{|c|}{$\mathrm{T}_{\mathrm{m}}(\mathrm{K})$} \\
\hline sDNA & $361.3 \pm 1.1$ \\
\hline sDNA/[BMIM]TfO & $360.9 \pm 0.7$ \\
\hline sDNA/[MIM]TfO & $360.4 \pm 1.2$ \\
\hline sDNA/[BMIM]MeSO & $352.9 \pm 1.6$ \\
\hline
\end{tabular}

It is noteworthy that the value of $\mathrm{T}_{\mathrm{m}}=361.3 \pm 1.1 \mathrm{~K}$ obtained for sDNA is in good agreement with the temperature of melting point predicted by UV absorption and DSC measurements (see next sections). This further confirms that the Raman intensity of dG-I band is mainly sensitive to the final step of the unfolding process of DNA. As stated above, the values in Table 1 indicate a remarkable decrement of $\mathrm{T}_{\mathrm{m}}$ observed for the case of DNA in the presence of [BMIM] $\mathrm{MeSO}_{4}$. This could be sketched assuming that the imidazolium cation tends to interact mainly with the DNA backbone when the negative charges of phosphate groups are localized [20,22], and the anion $\mathrm{MeSO}_{4}{ }^{-}$tends to establish $\mathrm{H}$-bond interactions with the acceptor/donor sites of guanine 
bases present on the major and minor grooves of DNA [17]. Overall, these interactions established between [BMIM] $\mathrm{MeSO}_{4}$ and the structure of DNA affect the thermal unfolding process resulting in the observed decrement of the $T_{m}$ of the system. The data of Table 1 show that the effect of $\mathrm{TfO}^{-}$anion on $\mathrm{T}_{\mathrm{m}}$ is negligible compared to that of $\mathrm{MeSO}_{4}{ }^{-}$, keeping constant the BMIM cation. This is probably due to the stronger hydrogen bond acceptor (HBA) character of $\mathrm{MeSO}_{4}{ }^{-}$with respect to $\mathrm{TfO}^{-}$. A rationale of this can be found in the opposite electronic effect of the two substituents $\left(\mathrm{CF}_{3}\right.$ and $\mathrm{OCH}_{3}$ functional groups) at the same $\mathrm{SO}_{3}{ }^{-}$polar head in $\mathrm{TfO}^{-}$ and $\mathrm{MeSO}_{4}{ }^{-}$, respectively (see Figure 1 ). The $\mathrm{CF}_{3}$ group has a strong - I effect (inductive electron withdrawing effect) whereas $\mathrm{OCH}_{3}$ is a strong resonance electron donor (+M effect). As shown, the HBA strength is enhanced by the presence of electron donating groups. [S. J. Pike, J. J. Hutchinson, C. A Hunter, H-bond acceptor parameters for anions, J. Am. Chem. Soc. 193 (2017) 6700-6706 https://doi.org/10.1021/jacs.17b02008]

Pre-melting structural changes on $d A-d T$ base pairs

The analysis of the UVRR spectra collected using $266 \mathrm{~nm}$ as excitation wavelength provides a special focus on the adenine response during the thermal unfolding path of sDNA. Figure 5 shows the temperature-dependence of the relative intensity of the mode dA-I at $\sim 1339 \mathrm{~cm}^{-1}$ for sDNA in absence and presence of ILs.

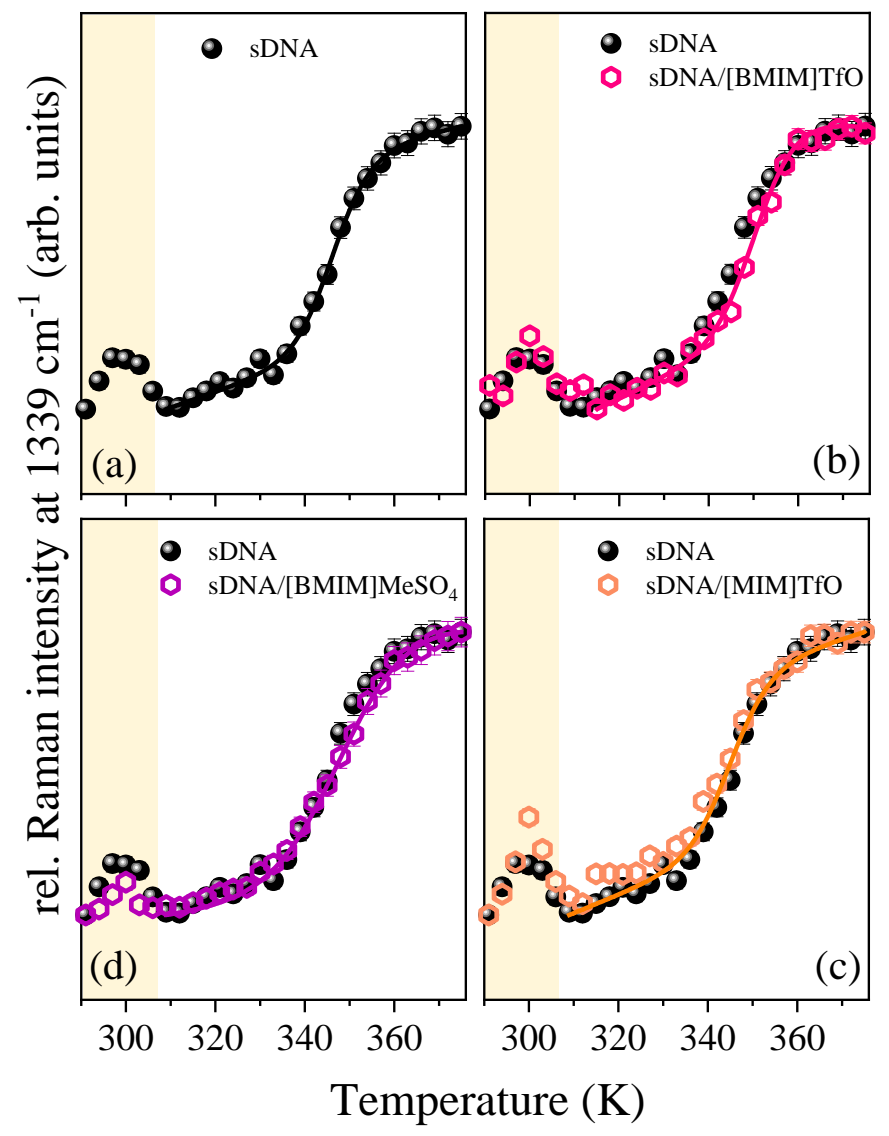

Figure 5: Temperature-dependent relative UVRR intensities of dA-I at $\sim 1339 \mathrm{~cm}^{-1}$ for sDNA (a) and sDNA in the presence of [BMIM]TfO (b), [MIM]TfO (c) and [BMIM] $\mathrm{MeSO}_{4}(\mathrm{~d})$. Continuous lines are fitting of the experimental data by using eqn. (1), see details in the text. The colored area highlights the temperature range where the pre-melting process is detected. 
The intensity of band dA-I for sDNA clearly exhibits a non-monotonic dependence upon temperature. We can distinguish three different temperature regimes, i.e. a premelting and melting region in which the resonance Raman intensity of dA-I displays a remarkable temperature dependence and a post-melting regime for $\mathrm{T} \gtrsim 360 \mathrm{~K}$ where no significant changes are observed in the Raman intensity. Compared to the behavior shown for dG-I band in Fig. 4, data of Fig. 5 suggest that dA-I mode is a marker of localized (non-cooperative) structural changes occurring on adenine residues at different stages of the thermal unfolding pathway of DNA. The pronounced peak in the Raman intensity of the dA-I band observed at approximately 300-303 K can be attributed to a specific pre-melting transition of the sDNA primarily localized on the dA-dT base pairs. Similar pre-melting phenomenon have been previously observed at temperature around 303-308 $\mathrm{K}$ for Poly $(\mathrm{dA}-\mathrm{dT}) \cdot \operatorname{poly}(\mathrm{dA}-\mathrm{dT})$ double-helical B DNA containing AT and TA base pairs [37,46,47] and in the genomic Calf thymus DNA [52]. These structural modifications have been explained by invoking the formation, at low temperature, of a third hydrogen bond cross-strand between consecutive dA - dT pairs that could stabilize the extra amount of propeller twist between the A-T base planes. The finding of a pre-melting transition in the plots of Fig. 5 suggests the occurrence of thermally activated structural changes in which the interbase A-T hydrogen bonding and the conformations of the duplex phosphodiester backbone of DNA are perturbed but neither significant unstacking nor dissociation of the double helix occur. The marked sensitivity of dA-I to non-cooperative structural changes can be explained with that fact that the vibration associated to this band is mainly localized on C5-N7=C8 bond of adenine where N7 atom is able to form HB with the solvent molecules [36,38,40,53]. At variance with DNA melting, pre-melting transitions reflect temperature-dependent conformational changes of small regions of the DNA molecule that are more effectively detected by A-T than C$\mathrm{G}$ base-pairs [54]. This is also consistent with theoretical calculations [55] that find an average value for $\mathrm{HB}$ lifetime on $\mathrm{N7}$ site of $\mathrm{dA}$ shorter with respect to the $\mathrm{HB}$ involving N7 of dG residue.

Quite interestingly, the plots in Fig. 5 point out that all the ILs considered in this study do not induce any significant effect on the pre-melting transition of sDNA. Since these structural changes involve primarily the base of adenine, our experimental finding is fully consistent with the simulation results [20] that indicate the dominant interaction of imidazolium cations with guanine and thymine on the strands of DNA.

In Fig. 5, the recovery of Raman hyperchromicity of dA-I band observed at about 340-350 K reflects the progressive unstacking of $\mathrm{dA}$ bases flanking the dissociation of the DNA strands occurring near the melting temperature. Above $\sim 360 \mathrm{~K}$ the intensity of dA-I band does not show appreciable variations. This is consistent with the breaking of inter-base Watson-Crick hydrogen bonds after the melting and dissociation of the sDNA strands. No further significant structural changes involving adenine bases occur at higher temperatures (post-melting region).

The two-step model of eqn. (1) can be reasonably used also for fitting the temperaturedependence of Raman intensity of dA-I between 310 and $375 \mathrm{~K}$ in Fig. 5, in order to determine the specific contribution of $\mathrm{dA}$ to the unfolding process of DNA [51]. Table 2 reports the values obtained for $\mathrm{T}_{\mathrm{m}}$ and $\Delta \mathrm{H}_{\mathrm{vib}}$ for DNA in absence and presence of ILs.

Table 2: Thermodynamic parameters estimated for the structural changes involving dA tracts for sDNA in presence and absence of ILs.

\begin{tabular}{|l|l|l|}
\hline & \multicolumn{1}{|c|}{$\mathrm{T}_{\mathrm{m}}(\mathrm{K})$} & \multicolumn{1}{c|}{$\Delta \mathrm{H}_{\mathrm{vib}}(\mathrm{kJ} / \mathrm{mol})$} \\
\hline sDNA & $345.9 \pm 1.3$ & $200.13 \pm 41.45$ \\
\hline sDNA/[BMIM]TfO & $349.4 \pm 1.2$ & $183.38 \pm 29.31$ \\
\hline sDNA/[MIM]TfO & $344.8 \pm 1.6$ & $183.8 \pm 46.89$ \\
\hline sDNA/[BMIM] $\mathrm{MeSO}_{4}$ & $347.3 \pm 1.1$ & $126.44 \pm 10.86$ \\
\hline
\end{tabular}


The transition temperature of 345-350 K obtained for all the DNA systems reflects the presence of a thermal intermediate state, occurring before the melting transition, that primarily involves dA-dT tracts in the DNA sequence. We can argue that this structural modification involves the progressive rupture of $\mathrm{HB}$ on the $\mathrm{dA}-\mathrm{dT}$ base pairs occurring at lower $\mathrm{T}$ than the melting temperature associated to the complete separation of the double strand of DNA. It is interesting to note that the enthalpy variation associated to this intermediate structural change is slightly lower for DNA in the case of presence of [BMIM] $\mathrm{MeSO}_{4}$.

Hydrogen-bonds rearrangements at $d G, d A$ and $d T$ sites

Figure 6(a)-(b) display the temperature-dependence of the wavenumber position of the bands dG-I and dA-I for sDNA in presence and absence of ILs. The frequency of the oscillators associated to dG-I $\sim 1482 \mathrm{~cm}^{-1}$ and dA-I $\sim 1339 \mathrm{~cm}^{-1}$ is sensitive to the different hydrogen bonding states on the proton-acceptor N7 site of guanine and adenine residues, respectively [36$38,40,41,56]$.

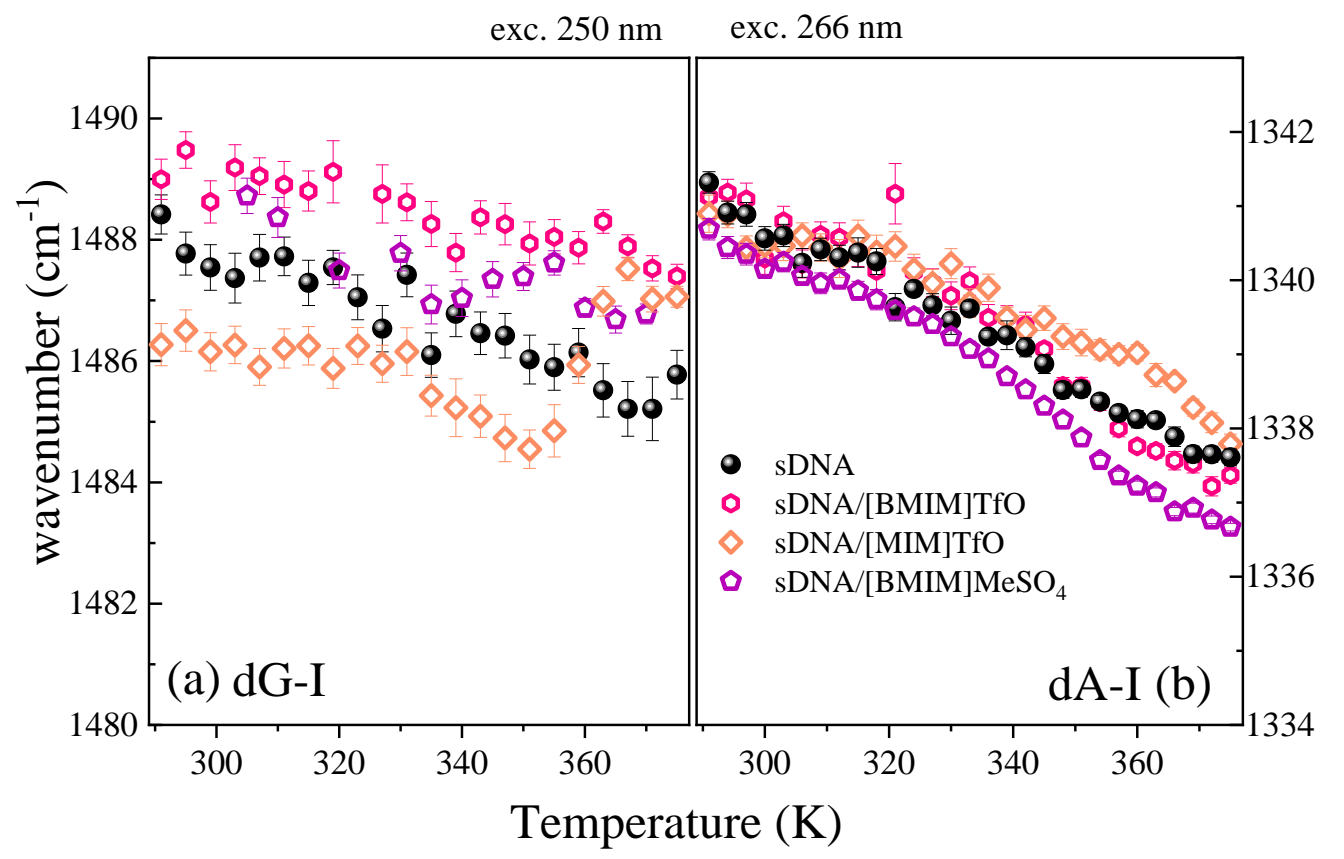

Figure 6: Temperature-dependent wavenumber position of the modes dG-I (a) and dA-I (b) for sDNA in absence and presence of [BMIM]TfO, [MIM]TfO and [BMIM] $\mathrm{MeSO}_{4}$.

Fig. 6(a) point outs a red-shift upon the increment of temperature of the dG-I mode that has been attributed to the formation of stronger hydrogen bonding of solvent with the guanine N7 site, occurring in the denatured structure of DNA [40,57]. We note that the presence of [BMIM] $\mathrm{MeSO}_{4}$ and [BMIM]TfO induces a slight blue-shift in the position of band dG-I with respect to the case of pristine DNA. This effect has been likewise ascribed to i) the formation of $\mathrm{H}$-bond interaction on $\mathrm{N7}$ atom of guanine with $\mathrm{CH}$ groups of [BMIM] and ii) the reduction of $\mathrm{H}$-bond interaction between water molecules and N7 site due to the presence of cations of IL [57,58]. The overall effect is consistent with the partial "dehydration” of DNA by ILs, predicted also by theoretical simulations [20, 22], that tend to hamper the diffusion of water molecules across the nucleobases in DNA. Fig. 6(a) points out also a peculiar behaviour for $\mathrm{T} \gtrsim 350 \mathrm{~K}$ for dG-I band in the presence of [MIM]TfO. This could be a marker of the trading base-base 
hydrogen bonds for the base-water hydrogen bonds starting close to the thermal denaturation and strongly affected by the presence of this type of ionic liquid.

The red-shift of the mode dA-I displayed in Fig. 6(b) for sDNA reflects the progressive weakening of $\mathrm{H}$-bonding at the acceptor sites $\mathrm{N} 7$ of adenine upon the increment of temperature $[37,38]$. This could be due to the replacement of inter-adenine hydrogen bonds with weaker adenine-water hydrogen bonds favored by the progressive exposition to the solvent of the residues of sDNA during the melting. Another possible explanation is that the reduction of the frequency of dA-I band could be related to the conformational change of dA residues from C2'endo to C3'-endo that accompanies the pre-melting and melting transition of the polyribonucleotide $[35,37,59]$. No significant modifications of the dA-I frequency induced by the presence of ILs are detected. This is consistent with a larger interaction of imidazolium cations with guanine with respect to adenine in the DNA structure, as discussed above.

Figures 7(a)-(b) report the frequency variations of the dG-II and dT bands as a function of temperature. Differently from the trend observed for dG-I and dA-I mode, the plots in Fig. 7 show a characteristic sigmodal shape with a general blue-shift with increasing temperature.

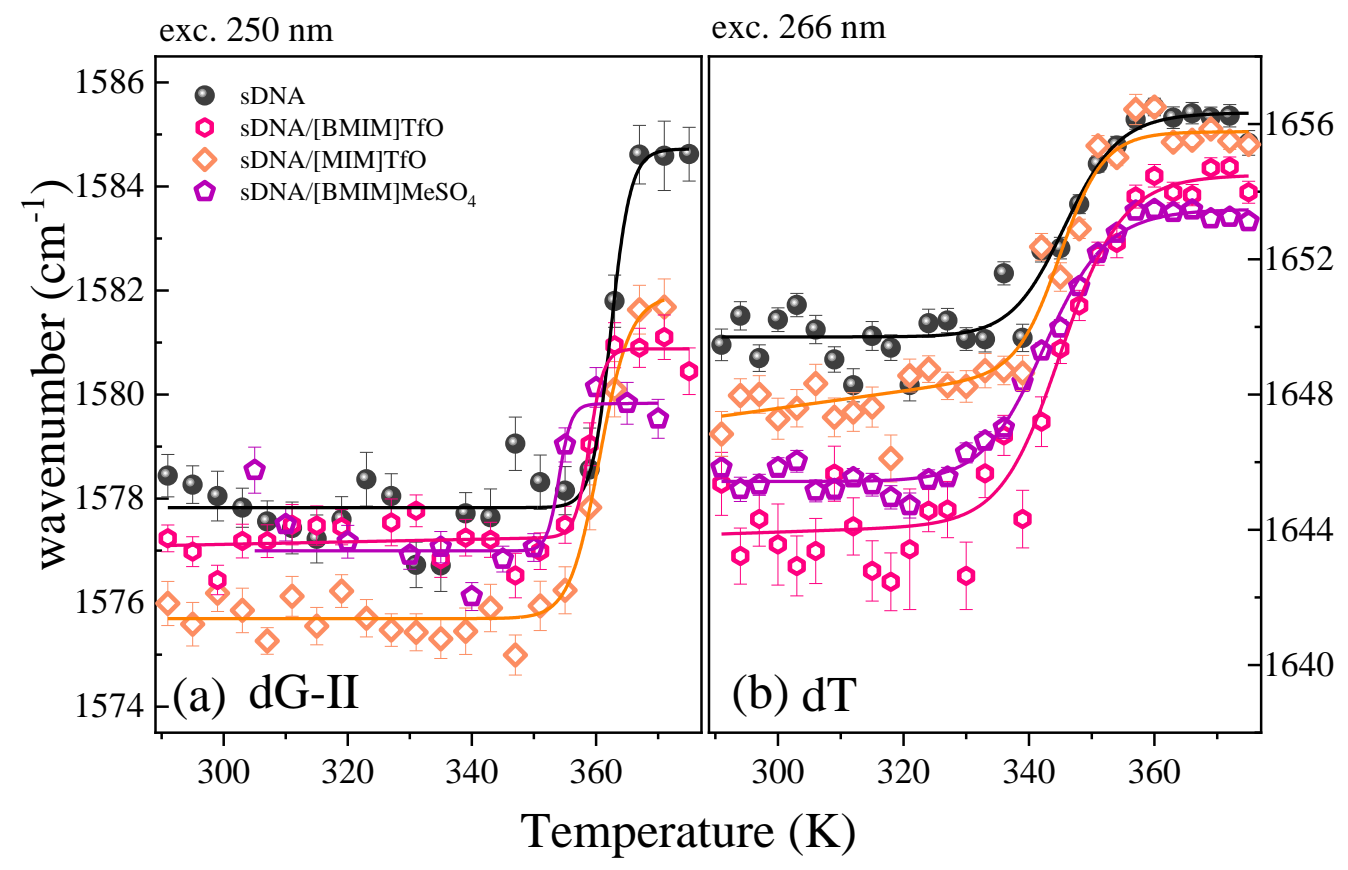

Figure 7: Temperature-dependent wavenumber position for the bands dG-II (a) and dT (b) for sDNA in absence and presence of [BMIM]TfO, [MIM]TfO and [BMIM] $\mathrm{MeSO}_{4}$. Continuous lines are fitting of the experimental data by using eqn. (1), see details in the text.

The dG-II band is attributed to stretching vibrational motions localized on the purine ring of guanine. Figure 7(a) shows that a rapid blue-shift in the frequency position of this mode is observed around the melting temperature $\mathrm{T}_{\mathrm{m}} \sim 360 \mathrm{~K}$. Fitting of the experimental data with the equation (1) gives indication of $\mathrm{T}_{\mathrm{m}}$ decrease of about $5 \mathrm{~K}$ in the case of sDNA in the presence of $[\mathrm{BMIM}] \mathrm{MeSO}_{4}$, consistently with the behavior observed for the intensity of dG-I band. The upshifts detected for dG-II band as a function of temperature are close related to hyperchromic effect previously discussed in the case of dG-I band (Fig. 4). This in turn reflects the unstacking effect between guanine bases generated by the increase of the temperature. Indeed, the 
reinforcement of the double bonds of the guanine ring is due to the major localization of the electrons within the purine structure in the single strand compared to the double strand.

Fig. 7(b) shows the temperature-dependence of the wavenumber position for $\mathrm{dT}$ band. This vibration accounts for any perturbations occurring at $\mathrm{C} 4=\mathrm{O}$ site of $\mathrm{dT}$ residue [30,38,47]. It is a marker of the decrease of H-bond strength at this site during the thermal unfolding pathway of sDNA. The trend in Fig. 7(b) confirms that the increase of thermal motion induces a general reduction of base stacking interactions and $\mathrm{H}$-bonding strength of the $\mathrm{dA}$ and $\mathrm{dT}$ residues in sDNA. It has the characteristic profile exhibited by dT band with a sharp upshift at about 345$350 \mathrm{~K}$, as suggested by the values of $\mathrm{T}_{\mathrm{m}}$ obtained by fitting of data with eqn (1). This could suggest a cooperative mechanism of the rearrangement of $\mathrm{H}$-bonding interactions at the $\mathrm{C} 4=\mathrm{O}$ site of thymine activated at temperature above 345-350 K. This is consistent with the formation of a three-centered H-bond in AT tracts, as already proposed by other authors [47]. We also note that the addition of ILs tends to slightly shift to lower frequency wavenumber the position of $\mathrm{dT}$ with respect to pristine sDNA, especially at low temperature. This supports the idea of a perturbation induced by ILs on the hydration shell of $\mathrm{C} 4=\mathrm{O}$ group of thymine with the formation of HB bonds with slightly higher strength, in agreement with the theoretical findings of A. Chandran et al. [20].

\section{UV absorption measurements}

In order to complement the UVRR data, UV absorption measurements on solution of sDNA in absence and presence of [BMIM] $\mathrm{MeSO}_{4}$, as an example, have been performed. Figure 8 shows the temperature evolution of the maximum of the intensity of absorption curve at $\sim 260 \mathrm{~nm}$ for sDNA with and without the addition of IL.

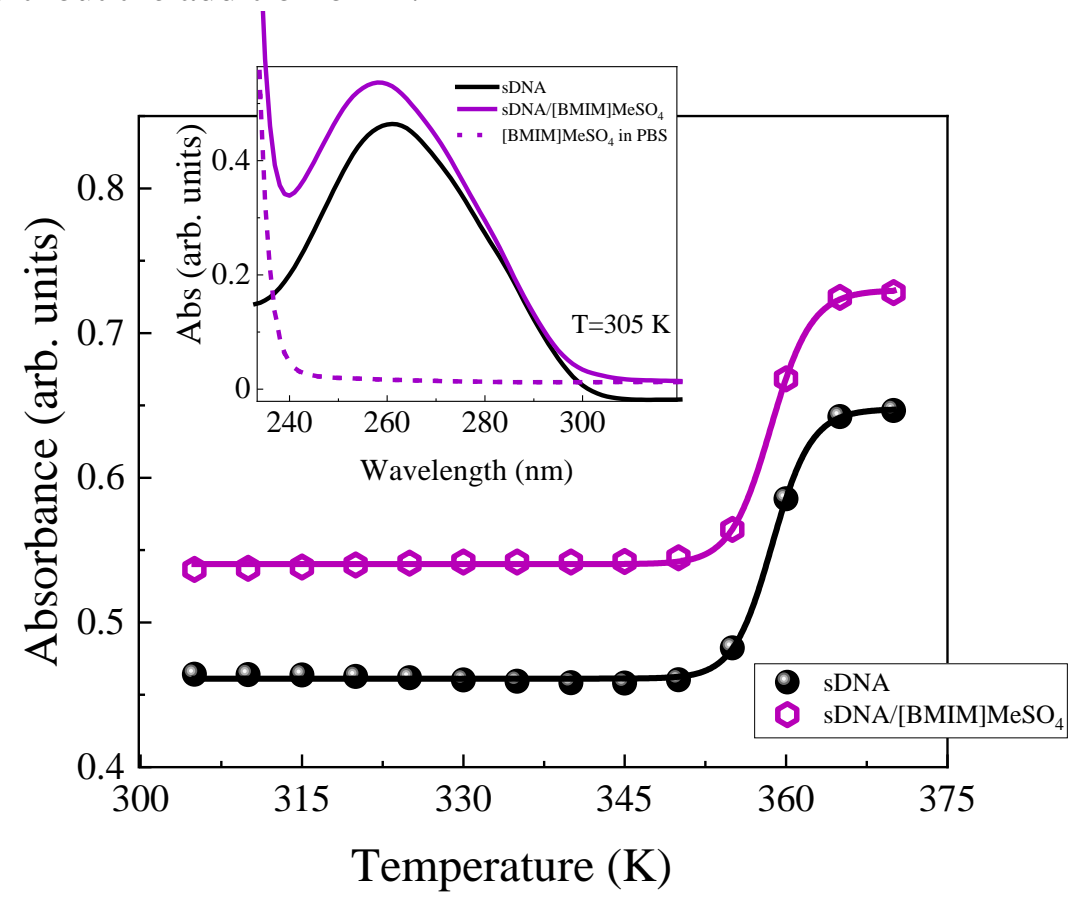

Figure 8: Temperature-dependent absorption at $260 \mathrm{~nm}$ for sDNA in absence and presence of $[\mathrm{BMIM}] \mathrm{MeSO}_{4}$. Inset: comparison between the UV absorption spectra of sDNA and sDNA/[BMIM] $\mathrm{MeSO}_{4}$ at $305 \mathrm{~K}$; the dashed line represents the UV absorption of $[\mathrm{BMIM}] \mathrm{MeSO}_{4}$ in water.

Since the absorption profile of pure [BMIM] $\mathrm{MeSO}_{4}$ is practically negligible up to $\sim 240 \mathrm{~nm}$, the data reported in Fig. 8 take account only of the contribution arising from DNA. As first remark, a 
sharp transition in the absorption intensity can be detected in correspondence of about $360 \mathrm{~K}$ for both the set of data, in the presence and absence of IL. This rapid increase of the UV absorption is a marker of the occurring of DNA melting and the melting temperature deduced by UV absorption technique for sDNA (359 $\pm 1 \mathrm{~K}$ ) is in agreement with that found by the analysis of UVRR spectra discussed above. The plots in Fig. 8 give also evidence that the UV absorption does not detect any base unstacking process until the melting temperature at about $360 \mathrm{~K}$.

\section{CD spectra}

By using CD technique, we can probe the temperature-dependent changes of geometry for DNA pure and in the presence of IL through the analysis of spectral modifications of CD spectra [60]. The CD measurements have been carried out on sDNA in absence and presence of [BMIM] $\mathrm{MeSO}_{4}$ as example. Figure 9(a) shows a comparison between the CD spectra of sDNA in absence and presence of IL collected at $305 \mathrm{~K}$.
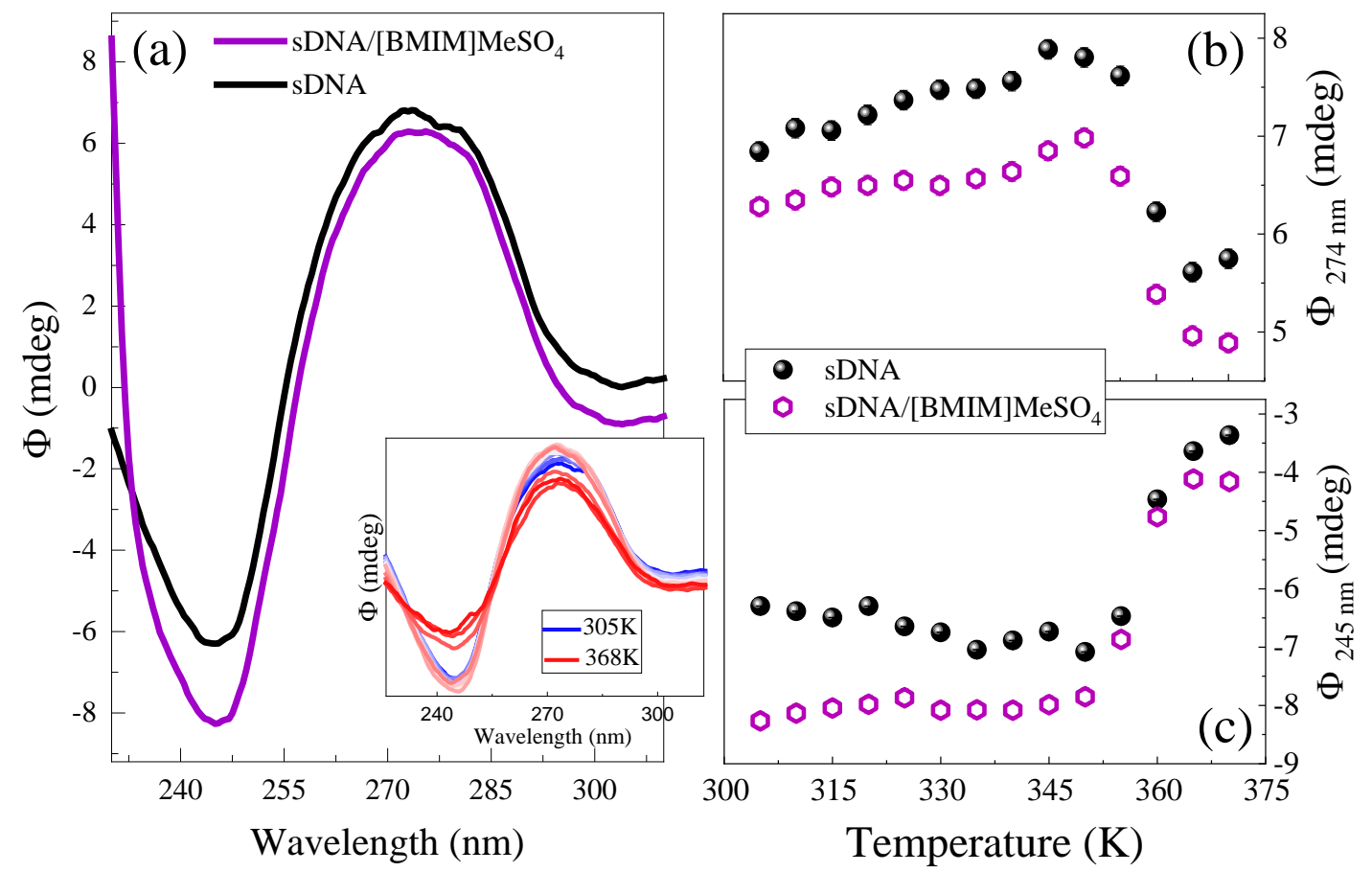

Figure 9: (a) Comparison between CD spectra of sDNA in absence and presence of [BMIM] $\mathrm{MeSO}_{4}$ measured at 305K. Inset: temperature-dependent CD spectra of pristine sDNA. Temperature-dependence of ellipticity at $274 \mathrm{~nm}$ (b) and $245 \mathrm{~nm}$ (c) for sDNA and sDNA/[BMIM]MeSO .

Since [BMIM] $\mathrm{MeSO}_{4}$ is an achiral compound, it does not exhibit any interfering CD band in the spectra of sDNA in hydrated IL. As shown Fig. 9(a), the CD signal of sDNA exhibits the classical features of double-stranded B-form DNA consisting of a positive band $\beta$ around $274 \mathrm{~nm}$ and a negative peak $\alpha$ at $245 \mathrm{~nm}$ with a crossover at the absorption maximum. The positive feature is due to $\pi-\pi$ base stacking while the negative band corresponds to the polynucleotide helicity. The comparison between the two curves in Fig. 9(a) evidences that the CD spectra of sDNA in presence and in absence of IL have a similar shape, thus suggesting that the native Bconformation of DNA is preserved also after the addition of [BMIM] MeSO 4 . This finding is in agreement with what already concluded by the analysis of UVRR data (see discussion above). By looking to the curves in Fig. 9(a), it clearly appears that the presence of [BMIM] $\mathrm{MeSO}_{4}$ 
induces a slight decrease in the positive band at about $274 \mathrm{~nm}$ of DNA. This is consistent with an alteration in the base packing, probably due to the hydrophobic interaction between the hydrocarbon chains of [BMIM] ${ }^{+}$and the bases of DNA [21]. It is also to noteworthy a significant decrease in the molar ellipticity that change from -6.3 to -8.3 when sDNA is in hydrated IL with respect to water. Such behavior reflects the distortion in the helical structure of B-DNA and could be related to a transition from an extended double helix to a more compact form, known as $\Psi$ structure, which is probably due to the electrostatic interaction between the cationic groups of IL and the phosphate groups of DNA. The absence in the CD spectra of sDNA/[BMIM]MeSO 4 of the typical signals associated to intercalation phenomena (magnitude of positive and negative bands) gives indication that IL does not act as an intercalator in DNA structure [61], consistently with the results of molecular dynamic simulations [20]. On the basis of the results reported in Fig. 9(a), we can speculate that $[\mathrm{BMIM}] \mathrm{MeSO}_{4}$ interacts with sDNA bases through groove and hydrophobic interactions, as found for the cases of [BMIM]Cl [20] and [BMIM]Br [22].

The temperature-dependent CD spectra of sDNA are reported in the inset of Fig. 9(a). Starting from the temperature of about $360 \mathrm{~K}$, the CD spectra clearly exhibit the characteristic shape associated to denatured DNA [62]. A decreasing in magnitudes of both the positive and negative bands is found in the CD spectra of pure DNA as the temperature increases. This is consistent with the occurrence of unstacking on the bases that leads to a general weakening of the interaction between neighboring bases and a decrease in helicity of DNA. This finding further confirms the interpretation of UVRR data discussed above.

The dependence on temperature of the $\beta$ intensity for DNA in presence and absence of IL is illustrated in Fig. 9(b). In the region of low temperature (305-350K), the ellipticity tends to increase upon $\mathrm{T}$ and this behavior suggests that $\mathrm{B} \rightarrow \mathrm{C}$ conformational changes of DNA take place in the premelting region [62]. For temperature values above about $350 \mathrm{~K}$, we observe an opposite temperature-dependent trend in the ellipticity, probably due to the beginning of denaturation process. Likewise, Fig. 9(c) shows the temperature evolution of $\alpha$ band. As mentioned above, modifications occurring in ellipticity in such wavelength region can be associated to changes in helicity, which are characteristic for the right-handed B form of DNA. The sigmoidal shape displayed in Fig. 9(c) strictly resembles that obtained for the UV-Vis absorbance (see Figure 8).

mDSC results

As for UV absorption and CD measurements, mDSC analysis was performed on sDNA in absence and presence of $[\mathrm{BMIM}] \mathrm{MeSO}_{4}$, as a representative system. This analysis was carried out to validate the thermodynamic parameters, i.e. melting temperature and enthalpy, obtained from UVRR experiments and discussed above. Calorimetry, is a standard technique to collect information about thermal transitions of a material and it has been routinely employed for the calculation of the temperature-induced melting of nucleic acids [63,64]. Figure 10 shows the thermograms obtained from sDNA and sDNA/[BMIM] $\mathrm{MeSO}_{4}$ in the proximity of the melting transition. Melting transition can be detected as an endothermic event, from which the transition temperature can be obtained from the peak value or the onset. The calculated peak and onset values for sDNA alone were $360.8 \pm 0.1 \mathrm{~K}$ and $356.5 \pm 0.1 \mathrm{~K}$, respectively and are comparable with the melting temperature obtained from UVRR spectra (see Table 1). The thermograms in Fig. 10 point out that the adding of $[\mathrm{BMIM}] \mathrm{MeSO}_{4}$ induces a slightly decrease in the melting transition of DNA, as evidenced by the calculated peak and onset values (358.5 $\pm 0.4 \mathrm{~K}$ and 353.5 
$\pm 0.4 \mathrm{~K}$, respectively) and in agreement with UVRR findings (see data reported in Table 1). As regards the $\Delta \mathrm{H}$ enthalpy associated to the calorimetric transition, the calculated values indicate a slight increment of the $\Delta \mathrm{H}$ associated to the melting process of DNA in the presence of [BMIM] $\mathrm{MeSO}_{4}(412.4 \pm 71.2$ and $450.9 \pm 82.9 \mathrm{~kJ} / \mathrm{mol}$ for sDNA and sDNA/[BMIM]MeSO 4 , respectively).

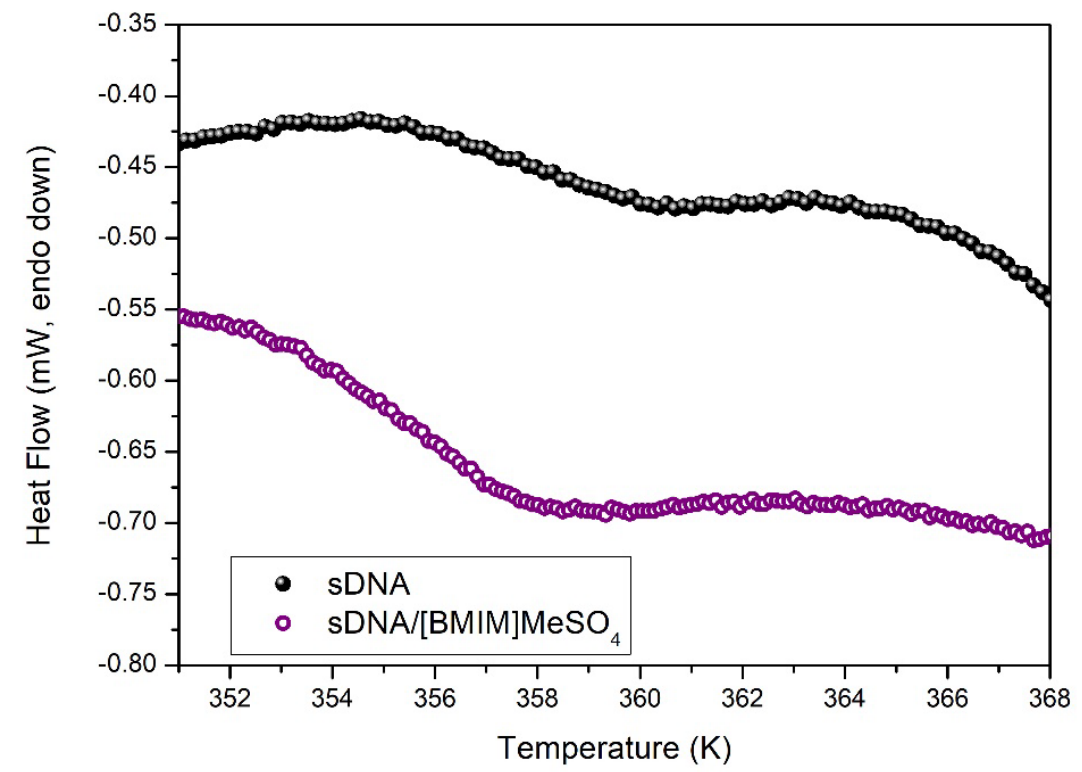

Figure 10: Thermograms of sDNA in absence and presence of [BMIM] $\mathrm{MeSO}_{4}$ in the vicinity of the melting transition.

\section{Summary and conclusions}

The molecular interactions and the effect of imidazolium-based ILs on the thermal unfolding process of DNA are here discussed on the basis of the results provided by synchrotron-based UVRR experiments. Our experimental approach benefits to afford a selective focus on the guanine and adenine responses respect to those of other components of the DNA structure, whose Raman signal may be strongly enhanced in intensity trough an accurate and fine choice of the excitation wavelength. The UVRR spectra of DNA collected using 250 and $266 \mathrm{~nm}$ as excitation wavelengths selectively probe two distinct temperature-dependent phenomena involving the structural rearrangement of the DNA double-helix: i) non-cooperative pre-melting transitions occurring throughout the range 300-350 K which essentially affect $\mathrm{dA}$ tracts, involving neither dissociation of the strands of DNA nor significant base unstacking; ii) a cooperative melting transition, observed in the range $360-370 \mathrm{~K}$, that is characterized by unstacking interactions between the guanine bases and strand separation. The analysis of the UVRR spectra of DNA in absence and presence of ILs reveals that both cations and anions of IL strongly interact with the structure of DNA, thus affecting the melting process. This may be due to establishment of H-bond interactions between cation/anion of ILs and the acceptor/donor sites of guanine bases present on the major and minor grooves of DNA, in accordance with theoretical simulations and previous experimental results [17,20,22]. Despite the formation of a strong interaction between ILs and the structure of DNA, the pre-melting conformations that precede the double-strand separation are not perturbed by the presence of ILs. This experimental finding 
supports the conclusion of the theoretical work of Chandran et al. [20] that suggest a dominant interaction of imidazolium cation with guanine and thymine on the strands of DNA, with respect to adenine.

The main results achieved by the analysis of UVRR spectra are validated by the information provided by UV absorption, circular dichroism and calorimetry that corroborate the reliability of our experimental approach. The overall results lead to a comprehensive view of the interactions between imidazolium-based ILs and DNA that provide important basis for the application of ILs in life science.

\section{References}

[1] R. Seddon, Ionic Liquids for Clean Technology, J. Chem. Tech. Biotechnol. 68 (1997) 351356 https://doi.org/10.1002/(SICI)1097-4660(199704)68:4<351::AID-JCTB613>3.0.CO;2-4 [2] Z. Lei, B. Chen, Yoon-Mo Koo, D. R. MacFarlane, Introduction: Ionic Liquids, Chem. Rev. 117(10) (2017) 6633-6635 https://doi.org/10.1021/acs.chemrev.7b00246

[3] J. P. Hallett, T. Welton, Room-Temperature Ionic Liquids: Solvents for Synthesis and Catalysis. 2, Chem. Rev. 111(5) (2011) 3508-3576 https://doi.org/10.1021/cr1003248

[4] D. R. MacFarlane, J. M. Pringle, P. C. Howlett, M. Forsyth, Ionic liquids and reactions at the electrochemical interface, Phys. Chem. Chem. Phys. 12 (2010) 1659-1669 https://doi.org/10.1039/B923053J

[5] X. Shen, B. Sun, F. Yan, J. Zhao, F. Zhang, S. Wang, X. Zhu, S. Lee, High-Performance Photoelectrochemical Cells from Ionic Liquid Electrolyte in Methyl-Terminated Silicon Nanowire Arrays, ACS Nano 4(10) (2010) 5869-5876 https://doi.org/10.1021/nn101980x

[6] W. Xu, C. A. Angel, Science 302 (2003) 422-425 https://doi.org/10.1126/science.1090287

[7] T. Welton, Coord. Chem. Rev. $248 \quad$ (2004) 2459-2477 https://doi.org/10.1016/j.ccr.2004.04.015

[8] M. Armand, F. Endres, D. R. MacFarlane, H. Ohno, B. Scrosati, Ionic-liquid materials for the electrochemical challenges of the future, Nat. Mater. 8 (2009) 621-629 https://doi.org/10.1038/nmat2448

[9] T. Welton, Room-Temperature Ionic Liquids. Solvents for Synthesis and Catalysis, Chem. Rev. 99 (1999) 2071-2084 https://doi.org/10.1021/cr980032t

[10] M. Erbeldinger, A.J. Mesiano, A.J. Russell, Enzymatic catalysis of formation of zaspartame in ionic liquid-an alternative to enzymatic catalysis in organic solvents, Biotechnology Progress 16 (2000) 1129-1131 https://doi.org/10.1021/bp000094g

[11] A. Benedetto, P. Ballone, Room Temperature Ionic Liquids Meet Biomolecules: A Microscopic View of Structure and Dynamics, ACS Sustainable Chem. Eng. 4 (2016) 392-412 https://doi.org/10.1021/acssuschemeng.5b01385

[12] K. S. Egorova, E. G. Gordeev, V. P. Ananikov, Biological Activity of Ionic Liquids and Their Application in Pharmaceutics and Medicine, Chem. Rev. 117 (2017) 7132-7189 https://doi.org/10.1021/acs.chemrev.6b00562

[13] D. Saha, A. Mukherjee, Effect of water and ionic liquids on biomolecules, Biophys Rev. 10(3) (2018) 795-808 https://doi.org/10.1007/s12551-018-0399-2

[14] M. Sivapragasam, M. Moniruzzaman, M. Goto, Recent advances in exploiting ionic liquids for biomolecules: Solubility, stability and applications, Biotechnol. J. 11 (2016) 1000-1013 https://doi.org/10.1002/biot.201500603 
[15] R Vijayaraghavan, A. Izgorodin, V. Ganesh, M. Surianarayanan, D. R MacFarlaneet, Longterm structural and chemical stability of DNA in hydrated ionic liquids, Angew. Chem., Int. Ed. 49 (2010) 1631-1633 https://doi.org/10.1002/anie.200906610

[16] H. Tateishi-Karimata, N. Sugimoto, Structure, stability and behaviour of nucleic acids in ionic liquids, Nucleic Acids Research 42(14) (2014) 8831-8844 https://doi.org/10.1093/nar/gku499

[17] L. Cardoso, N. M. Micaelo, D NA molecular solvation in neat ionic liquids, Chem. Phys. Chem 12 (2011) 275-277 https://doi.org/10.1002/cphc.201000645

[18] S. Satpathi, A. Sengupta, V. M. Hridya, K.Gavvala, R. Kumar Koninti, B. Roy, P. Hazra, A Green Solvent Induced DNA Package, Scientific Reports 5(2015) 9137 https://doi.org/10.1038/srep09137

[19] N. Singh, M. Sharma, D. Mondal, M. M. Pereira, K. Prasad, Very High Concentration Solubility and Long-Term Stability of DNA in an Ammonium-Based Ionic Liquid: A Suitable Medium for Nucleic Acid Packaging and Preservation, ACS Sustainable Chem. Eng. 5 (2017) 1998-2005 https://doi.org/10.1021/acssuschemeng.6b02842

[20] A. Chandran, D. Ghoshdastidar, S. Senapati, Groove Binding Mechanism of Ionic Liquids: A Key Factor in Long-Term Stability of DNA in Hydrated Ionic Liquids, J. Am. Chem. Soc. 134 (2012) 20330-20339 https://doi.org/10.1021/ja304519d

[21] Y. Ding, L. Zhang, J. Xie, R. Guo, Binding Characteristics and Molecular Mechanism of Interaction between Ionic Liquid and DNA, J. Phys. Chem. B 114 (2010) 2033-2043 https://doi.org/10.1021/jp9104757

[22] K. Jumbri, M. B. Abdul Rahman, E. Abdulmalek, H. Ahmada, N. M. Micaelo, An insight into structure and stability of DNA in ionic liquids from molecular dynamics simulation and experimental studies, Phys. Chem. Chem. Phys. $16 \quad$ (2014) 14036-14046 https://doi.org/10.1039/C4CP01159G

[23] H. Liu, Y. Dong, J. Wu, C.Chen, D. Liu, Q. Zhang, S. Du, Evaluation of interaction between imidazolium-based chloride ionic liquids and calf thymus DNA, Science of the Total Environment 1-7 (2016) 566-567 https://doi.org/10.1016/j.scitotenv.2016.05.087

[24] P.K. Singh, J. Sujana, A. K. Mora, S. Nath, Probing the DNA-ionic liquid interaction using an ultrafast molecular rotor, Journal of Photochemistry and Photobiology A: Chemistry 246 (2012) 16- 22 http://dx.doi.org/10.1016/j.jphotochem.2012.07.006

[25] J. H. Wang, D. H. Cheng, X. W. Chen, Z. Du, Z. L. Fang, Direct extraction of doublestranded DNA into ionic liquid 1-butyl-3-methylimidazolium hexafluorophosphate and its quantification, Anal. Chem. 79 (2007) 620-625. http://dx.doi.org/10.1021/ac061145c

[26] K. Jumbri, H. Ahmad, E. Abdulmalek, M. Basyaruddin Abdul Rahman, Biophysical properties of DNA in hydrated ionic liquids, AIP Conference Proceedings 1787 (2016) 070003 https://doi.org/10.1063/1.4968137

[27] K. Jumbri, H. Ahmad, E.Abdulmalek, M. Basyaruddin, A. Rahman, Binding energy and biophysical properties of ionic liquid-DNA complex: Understanding the role of hydrophobic $\begin{array}{lllll}\text { interactions, } & \text { J. } & \text { Mol. } & \text { Liquid. } & 223\end{array}$ http://dx.doi.org/10.1016/j.molliq.2016.09.040

[28] B. Rossi, C. Bottari, S. Catalini, A. Gessini, F. D’Amico, C. Masciovecchio, Synchrotron based UV Resonant Raman scattering for material science, Molecular and Laser Spectroscopy, Volume 2 (eds V. P. Gupta, Y. Ozaki), Elesevier (2020), Chapter 13, pages 447-478, http://dx.doi.org/10.1016/B978-0-12-818870-5.00013-7 
[29] J. R. Perno, C. A. Grygon, T. G. Spiro, Ultraviolet Raman Excitation Profiles for the Nucleotides and for the Nucleic Acid Duplexes Poly(rA)-Poly(rU) and Poly(dG-dC), J. Phys. Chem. 93 (1989) 5672-5678 https://doi.org/10.1021/j100352a009

[30] S. P. A. Fodor, R. P. Rava, T. R. Hays, T. G. Spiro, Ultraviolet resonance Raman spectroscopy of the nucleotides with 266-, 240-, 218-, and 200-nm pulsed laser excitation, J. Am. Chem. Soc. 107 (1985) 1520-1529 https://doi.org/10.1021/ja00292a012

[31] S. P. A. Fodor, T. G. Spiro, Ultraviolet resonance Raman spectroscopy of DNA with 200266-nm laser excitation, J. Am. Chem. Soc. 108 (1986) 3198-3205 https://doi.org/10.1021/ja00272a006

[32] F. D'amico, M. Saito, F. Bencivenga, M. Marsi, A. Gessini, G. Camisasca, E. Principi, R. Cucini, S. Di Fonzo, A. Battistoni, E. Giangrisostomi, C. Masciovecchio, UV resonant Raman scattering facility at Elettra, Nucl. Instruments Methods Phys. Res. Sect. A Accel. Spectrometers, Detect. Assoc. Equip. 703 (2013) 33-37. https://doi.org/ 10.1016/j.nima.2012.11.037.

[33] I. Tinoco, Hypochromism in Polynucleotides, J. Am. Chem. Soc. 82 (1960) 4785-4790 https://doi.org/10.1021/ja01503a007

[34] Z. Q. Wen, G. J. Thomas Jr., UV resonance Raman spectroscopy of DNA and protein constituents of viruses: assignments and cross sections for excitations at 257, 244, 238, and 229 nm, Biopolymers 45(5) (1998) 247-256 https://doi.org/10.1002/(SICI)10970282(199803)45:3<247::AID-BIP7>3.0.CO;2-R.

[35] J. M. Benevides, S.A. Overman, G.J. Thomas Jr, Raman, polarized Raman and ultraviolet resonance Raman spectroscopy of nucleic acids and their Complexes, J. Raman Spectrosc. 36 (2005) 279-299 https://doi.org/10.1002/jrs.1324

[36] N. Fujimoto, A. Toyama, H. Takeuchi, Effects of hydrogen bonding on the UV resonance Raman bands of the adenine ring and its C8-deuterated analog, J. Mol. Structure 447 (1998) 6169 https://doi.org/10.1016/S0022-2860(98)00301-9

[37] L. Movileanu, J. M. Benevides, G. J. Thomas Jr, Temperature Dependence of the Raman Spectrum of DNA. Part I-Raman Signatures of Premelting and Melting Transitions of Poly(dA-dT) Poly(dA-dT), J. Raman Spectrosc. $30 \quad$ (1999) 637-649 https://doi.org/10.1002/(SICI)1097-4555(199908)30:8<637::AID-JRS431>3.0.CO;2-B

[38] A. Toyama, H. Takeuchi, I. Harada, Ultraviolet resonance Raman spectra of adenine, uracil and thymine derivatives in several solvents. Correlation between band frequencies and hydrogenbonding states of the nucleic acid bases, J. Mol. Structure 242 (1991) 87-98 https://doi.org/10.1016/0022-2860(91)87129-6

[39] S. Chan, R. H. Austin, I. Mukerji, T. G. Spiro, Temperature-Dependent Ultraviolet Resonance Raman Spectroscopy of the Premelting State of dA* dT DNA, Biophysical Journal 72 (1997) 1512-1520 https://doi.org/10.1016/S0006-3495(97)78799-X

[40] J. G. Duguid, V. A. Bloomfield, J. M. Benevides, G. J. Thomas, DNA melting investigated by differential scanning calorimetry and Raman spectroscopy., Biophysical Journal 71 (1996) 3350-3360 https://doi.org/10.1016/S0006-3495(96)79528-0

[41] J. G. Duguid, V.A. Bloomfield, J. M. Benevides, G. J. Thomas Jr., Raman Spectroscopy of DNA-Metal Complexes. I. The Thermal Denaturation of DNA in the Presence of Sr' , Ba2, Mg2+, Ca2+, Mn2+, C02+, Ni2+, and Cd2+, Biophysical Journal 69 (1995) 2623-2641 https://doi.org/10.1016/S0006-3495(95)80133-5

[42] P.Y. Turpin, L. Chinsky, A. Laigle, B. Jollès, DNA structure studies by resonance Raman spectroscopy, J. Mol. Structure 214 (1989) 43-70 https://doi.org/10.1016/0022-2860(89)80005-5 
[43] S. C. Erfurth, W. I. Peticolas, Melting and premelting phenomenon in DNA by laser Raman scattering, Biopolymers 14 (1975) 247-264 https://doi.org/10.1002/bip.1975.360140202

[44] W. Hug, I. Ticono, Electronic spectra of nucleic acid bases. I. Interpretation of the in-plane spectra with the aid of all valence electron MO-CIA [configuration interaction] calculations, J. Am. Chem. Soc. 95 (1973) 2803-2813 https://doi.org/10.1021/ja00790a010

[45] M. Tsuboi, M. Komatsu, J. Hoshi, E. Kawashima, T. Sekine, Y. Ishido, M. P. Russell, J. M. Benevides, G. J. Thomas, Raman and Infrared Spectra of (2`S)-[2`-2H]Thymidine: Vibrational Coupling between Deoxyribosyl and Thymine Moieties and Structural Implications, J. Am. Chem.Soc. 119 (1997) 2025-2032 https://doi.org/10.1021/ja962676t

[46] A. Jirasek, H. G. Schulze, C. Hughesman, A. L. Creagh, C. A. Haynes, M. W. Blades, R. F. B. Turner, Discrimination between UV radiation-induced and thermally induced spectral changes in AT-paired DNA oligomers using UV resonance Raman spectroscopy, J. Raman Spectrosc. 37 (2006)1368-1380 https://doi.org/10.1002/jrs.1552

[47] I. Mukerji, A. P. Williams, UV resonance Raman and circular dichroism studies of a DNA duplex containing an $\mathrm{A}(3) \mathrm{T}(3)$ tract: evidence for a premelting transition and three-centered $\mathrm{H}$ bonds, Biochemistry 41 (2002) 69-77 https://doi.org/10.1021/bi010918i

[48] L. Chinsky, P.Y.Turpin, Ultraviolet resonance Raman study of DNA and of its interaction with actinomycin D, Nucleic Acid Research 5(8) (1978) 2969-2978 https://doi.org/10.1093/nar/5.8.2969

[49] B. Tomlinson, W. L. Peticolas, Conformational Dependence of Raman Scattering Intensities in Polyadenylic Acid, J. Chem. Phys. 52 (1970) 2154 https://doi.org/10.1063/1.1673270

[50] F. Bianchi, L. Comez, R. Biehl, F. D’Amico, A. Gessini, A. M. Longo, C. Masciovecchio, C. Petrillo, A. Radulescu, B. Rossi, F. Sacchetti, F. Sebastiani, N. Violini, A. Paciaroni, Structure of human telomere G-quadruplex in the presence of a model drug along the thermal unfolding pathway, Nucleic Acids Research 46(22) (2018) 11927-11938 https://doi.org/10.1093/nar/gky1092

[51] L. Movileanu, J. M. Benevides, G. J. Thomas Jr, Determination of base and backbone contributions to the thermodynamics of premelting and melting transitions in B DNA, Nucleic Acids Res. 30 (17) (2002) 3767-3777 https://doi.org/10.1093/nar/gkf471

[52] L. Rimai, V. M. Maher, D. Gill, I. Salmeen, J. J. McCormick, The temperature dependence of Raman intensities of DNA. Evidence for premelting changes and correlations with ultraviolet spectra, Biochimica et Biophysica Acta, 361 (1974) 155-165 https://doi.org/10.1016/00052787(74)90343-8

[53] M. Shanmugasundaram, M. Puranik, Vibrational markers of structural distortion in adenine nucleobases upon DNA damage, Phys. Chem. Chem. Phys. 13 (2011) 3851-3862 https://doi.org/10.1039/c0cp01731k

[54] A. V. Lukashin, A. V. Vologodskii, M. D. Frank-Kamenetskii, Y. L. Lyubchenko, Fluctuational Opening of the Double Helix as Revealed by Theoretical and Experimental Study of D N A Interaction with Formaldehyde, J. Mol. Biol. 108 (1976) 665-682 https://doi.org/10.1016/s0022-2836(76)80111-8

[55] A. M. J. J. Bonvin, M. Sunnerhagen, G. O. Wilfred, F.van Gunsteren, Water molecules in DNA recognition II: a molecular dynamics view of the structure and hydration of the trp operator, J. Mol. Biol. 282 (1998) 859-873 https://doi.org/10.1006/jmbi.1998.2034

[56] A. Toyama, N. Fujimoto, N.i Hanada, J. Ono, E. Yoshimitsu, A. Matsubuchi, H. Takeuchi, J. Assignments and hydrogen bond sensitivities of UV resonance Raman bands of the C8deuterated 
guanine ring, J. Raman Spectrosc. 33 (2002) 699-708 https://doi.org/10.1002/jrs.899

[57] C. Bottari, I. Mancini, A. Mele, A. Gessini, C. Masciovecchio, B. Rossi, Conformational stability of DNA in hydrated ionic liquid by synchrotron-based UV resonance raman, Proc. SPIE 11086, UV and Higher Energy Photonics: From Materials to Applications 2019, 110860Q https://doi.org/10.1117/12.2529077

[58] J. G. Duguid, V. A., Bloomfield, J. M. Benevides, G. J. Thomas, Raman Spectroscopy of DNA-Metal Complexes. 1. Interactions and Conformational Effects of the Divalent Cations: $\mathrm{Mg}$, Ca, Sr, Ba, Mn, Co, Ni, Cu, Pd, and Cd," Biophys. J. 65 (1993) 1916-1928 https://doi.org/10.1016/S0006-3495(93)81263-3

[59] G. J. Thomas Jr., J. M. Benevides, An A Biopolymers 24 (1985) 1101-1105 https://doi.org/10.1002/bip.360240613

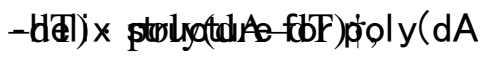

[60] D. S. Studdert, M. Patroni, R. C. Davis, Circular dichroism of DNA: temperature and salt dependence, Biopolymers 11 (1972) 761-779 https://doi.org/10.1002/bip.1972.360110404

[61] S. Parodi, F. Kendall, C. Nicolini, A clarification of the complex spectrum observed with the ultraviolet circular dichroism of ethidium bromide bound to DNA, Nucleic Acids Res., 2 (1975) 477-486 https://doi.org/10.1093/nar/2.4.477

[62] E. Zabost, A. Nowicka, M. Donten and Z. Stojek, Substantial difference between temperature dependencies of dsDNA predenaturation process obtained by voltammetry and spectroscopy, Phys. Chem. Chem. Phys. 11 (2009) 8933-8938 https://doi.org/10.1039/b906752c [63] C. Giancola, A convenient tool for studying the stability of proteins and nucleic acids: Differential scanning calorimetry, Journal of Thermal Analysis and Calorimetry 91 (2008) 1, 7985 https://doi.org/10.1007/s10973-007-8436-6

[64] D.S. Pilch, Calorimetry of Nucleic Acids Curr. Protoc. Nucleic Acid Chem. (2001) Chapter https://doi.org/10.1002/0471142700.nc0704s00 